\title{
SIMULTANEOUS APPROXIMATION WITH THE RAFU METHOD
}

\section{E. CORBACHO}

Abstract. Let $f$ be a function $k$ times continuously differentiable in $[a, b]$, then we will prove that the RAFU method provides a sequence $\left(H_{n}\right)_{n}$ defined in $[a, b]$ such that for each $j=0, \ldots, k$

$$
\left\|f^{(j)}-H_{n}^{(j)}\right\| \leqslant\left[\frac{M-m}{\sqrt{n}}+\omega\left(f^{(k)}, \frac{b-a}{n}\right)\right](b-a)^{k-j}
$$

being $n \geqslant 2,\|$.$\| the uniform norm, M$ and $m$ the maximum and the minimum of $f^{(k)}$ in $[a, b]$ respectively and $\omega\left(f^{(k)}, \frac{b-a}{n}\right)$ its modulus of continuity. The called RAFU remainder in Taylor's formula will be presented. The simultaneous approximation problem will be solved from average samples, from linear combinations, from local average samples given by convolution, from approximate values and in the case of non-uniformly spaced data of $f^{(k)}$. We will also study the numerical differentiation case. Our approach is easily realizable in computations. Some examples will be given.

Mathematics subject classification (2010): 41A28, 41A30, 37L65, 65D15.

Keywords and phrases: RAFU method, RAFU approximation, Simultaneous approximation.

\section{REFERENCES}

[1] E. Corbacho, Approximation in different smoothness spaces with the RAFU method, Appl. Gen. Topology, 15, 2 (2014), 221-228.

[2] E. Corbacho, A RAFU linear space uniformly dense in $C[a, b]$, Appl. Gen. Topology, 14, 1 (2013), $53-60$.

[3] E. CORBACHO, Uniform approximation with radical functions, $\mathrm{S} \vec{e}$ MA Journal, 58, (2012), 97-122.

[4] B. Della Vecchia, G. Mastroianni And P. VéRTesi, Simultaneous approximation by Hermite interpolation of higher order, J. Comput. Appl. Math., 50, (1994), 233-240.

[5] N. Hahm AND B. I. Hong, Simultaneous Approximation Algorithm Using a Feedforward Neural Network with a Single Hidden Layer, J. Korean Phys. Soc., 54, 6 (2009), 2219-2224.

[6] K. Kopotun, Simultaneous Approximation by Algebraic Polynomials, Constr. Approx., 12, (1996), 67-94.

[7] T. F. XIE AND F. L. CAO, The errors in simultaneous approximation by feed-forward neural networks, Neurocomputing, 73, (2010), 903-907. 